\title{
Stochastic Geometry Analysis of Throughput for Wireless Body Area Networks
}

\author{
Yinglong Wang, Ruixia Liu*, Minglei Shu and Changfang Chen
}

Shandong Computer Science Center (National Supercomputer Center in Jinan), Shandong Provincial Key Laboratory of Computer Networks, Jinan 250014, P.R. China

\begin{abstract}
Wireless Body Area Network (WBAN) coexistence is a very challenging problem, resulting in very strong interference among them, which seriously affects the reliability of communication. The interference is the main performance-limiting factor in wireless networks coexistence, and therefore it is crucial to mitigated. The goal of this paper is to decrease inter-WBANs interference and to increase the transmission success probability or throughput. One of the main determinants of the interference is the network geometry distribution. In this paper, the impact of channel sensing range to IEEE 802.15.4-based coexisting WBANs is analyzed and is optimized according to the nearest node's distance. In addition, we model the system adopting the stochastic geometry Poisson point process and analyze the impact of guard zone size to networks performance. Furthermore, the throughput efficiently achieves higher through optimizing the size of guard zone. Theoretical analysis and simulation results show that the reasonable setting of the protection area can improve effectively the system throughput, and the guard zone radius has an optimal value, which can make the throughput of the network to reach the maximum value. The energy and spectral efficiency, moreover, have been improved.
\end{abstract}

Keywords: WBANs coexistence; Stochastic geometry; Guard zone; Throughput; Success probability

\section{Introduction}

Population ageing is poised to become a global common concern social problem of the twenty-first century [1]. Wireless Body Area Network (WBAN) for health monitoring systems are expected to take an important role for health and the aged problems. In a health telemonitoring system, a WBAN is composed of a number of lightweight miniature sensors collecting vital signs, such as blood pressure, Electrocardiogram (ECG), body temperature etc. [2].

In many cases, several WBANs coexist in a small area, resulting in very strong interference among them, the loss of data and reducing networks reliability, which can lead to a serious threat to the patient's life. Therefore, WBANs coexistence is a very challenging problem. The interference is the main performance-limiting factor in wireless networks coexistence as well as increased power consumption and transmission latencies, and therefore it is crucial to mitigated.

On the other hand, stochastic geometry, in particular Poisson point process distribution, was already used in a variety of wireless network models the last decade.

Stochastic geometry provides a natural way of defining and computing critical performance metrics of the networks, such as the interference distribution, outage probability and so forth, by taking into account all potential geometrical patterns for the nodes, in the same way queuing theory provides response times or congestion, considering all potential arrival patterns [3].

Some Media Access Control (MAC) protocols create exclusion zones to protect scheduled transmissions. Aloha creates a random exclusion disc around each transmitter. By this we mean that for an arbitrary radius there is some non-null probability that all the nodes in the disk with this radius do not transmit at a given time slot. There will be at the same time a large enough random exclusion zone around any transmitter node [4].

In this paper, the impact of channel sensing range to IEEE 802.15.4-based coexisting WBANs is analyzed. In addition, we model the system adopting the stochastic geometry Poisson point process and analyze the impact of guard zone size to networks performance. Outage probabilities and coexisting network throughput are two important performance metrics that have been evaluated for certain specific types of wireless networks. Furthermore, the throughput efficiently achieves higher through optimizing the size of guard zone. Theoretical analysis and simulation results show that the reasonable setting of the protection area can effectively improve the system throughput, and the guard zone radius has an optimal value, which can make the throughput of the network to reach the maximum value. The energy and spectral efficiency, moreover, have been improved accordingly.

Our contributions are summarized as follows:

1. The impact of channel sensing range to IEEE 802.15.4-based coexisting WBANs is analyzed. By using stochastic geometry, we adjust the carrier sense range according to the closest node's distance.

2. The guard zone size of influence of networks coexist is analyzed, which can naturally decreases the interference and maximize the success probability. Furthermore, we tradeoff the throughput and the guard zone size of coexisting WBANs (Table 1).

\section{Related Work}

Some extensive efforts have been made to enhance the performance of WBANs coexistence. To avoid IEEE 802.11 interference, the authors

*Corresponding author: Ruixia Liu, Shandong Computer Science Center (National Supercomputer Center in Jinan), Shandong Provincial Key Laboratory of Computer Networks, Jinan 250014, P.R. China, Tel: 8653182605627; E-mail: liurx@ sdas.org

Received December 01, 2017; Accepted December 15, 2017; Published December 21, 2017

Citation: Wang Y, Liu R, Shu M, Chen C (2017) Stochastic Geometry Analysis of Throughput for Wireless Body Area Networks. Adv Robot Autom 6: 172. doi: 10.4172/2168-9695.1000172

Copyright: $\odot 2017$ Kumar S, et al. This is an open-access article distributed unde the terms of the Creative Commons Attribution License, which permits unrestricted use, distribution, and reproduction in any medium, provided the original author and source are credited. 


\begin{tabular}{|c|c|}
\hline Notation & Definition \\
\hline$\alpha$ & On-body path-loss exponent \\
\hline$h$ & Channel gain \\
\hline$\lambda_{p}$ & Intensity of WBANs \\
\hline & Carrier sense threshold \\
\hline$S$ & SIR threshold \\
\hline$P_{s}$ & Throughput \\
\hline$P_{t}$ & Transmission success probability \\
\hline & Transmission power value \\
\hline
\end{tabular}

Table 1: List of key notations.

proposed channel allocation algorithms that assigned channels which are less frequently used or unused by IEEE 802.11 devices to ZigBee sensors [5-7]. The authors used stochastic geometry analysis to develop a spectrum-efficient multi-channel random wireless networks [8]. By using graph theory, they obtained the minimum required number of channels to accommodate a certain intensity of coexisting networks under outage probability constraint and designed a superframe structure for the coexisting IEEE 802.15.4 networks and a method for time-domain interference alignment.

Many papers have already noticed the impact of carrier sense and spatial reuse on the system performance. The work indicates that virtual carrier sense is far from enough to solve the interference and larger physical carrier sense range can relieve to some extent [9]. In addition, there are many Matern Hard-Core Point Process (HCPP) model papers also. Some papers mainly modify model. A modified HCPP model is generalized for any fading environment [10]. Some papers analyzed interference. The authors obtained aggregate interference experienced by the test node by simulations based on the stochastic geometry [11].

In contrast, limited works have considered in WBANs. The previous works on WBANs mainly focused on transmission schemes to enhance the throughput. The purpose of this paper is to optimize the carrier sense and the guard zone to improve the system throughput.

\section{Modeling and the Success Probability Analysis}

Due to the distributed nature of WBANs coexistence, the MAClayer is responsible for coordinating channel accesses, by avoiding conflict and scheduling data transmissions, to maximize system throughput at an acceptable packet delay and minimal energy consumption. The 802.15.4 MAC, which serves a set of applications with very low power consumption and cost requirements, plays an important role and has a large impact on the performance of the WBANs [12].

An IEEE 802.15.4-based WBAN may be configured in the star, tree or mesh topology. Among them, the star topology is suitable for one hop wireless communication. Most of previously mentioned WBAN adopt this kind of structure because of short distance communication [13].

\section{The model of coexistence of IEEE802.15.4-based WBANs}

In order to provide an accurate and analytically convenient assumption for the node distribution, we model the coexistence of WBANs using the Poisson Point Process (PPP) model. The PPP model, which consists of randomly located points located on some underlying mathematical space, play a fundamental role in stochastic geometry. The simultaneous transmitters are modeled by homogeneous PPP in the network with density $\lambda_{p}$.

The location of WBANs transmitting nodes are modeled as a stationary and isotropic PPP $\varphi$ in the $\mathbb{R}^{2}$ Euclidean space illustrated in Figure 1. Each WBAN is composed of the Coordinator Node $(\mathrm{CN})$ and Terminal Nodes (TNs). All TNs are assumed that they have exactly the same performance and parameters. We consider a TN in each WBAN and a coordinator in this PPP model. In particular, let $d$ denotes the distance between node TN and CN. Then the received power $P_{r}$ at a distance $d$ from TN to $\mathrm{CN}$ is $P h d^{-\alpha}$, where the $\alpha$ is the path loss exponent and the random variable $h$ follows an exponential distribution with mean $1 / \mu$, which we denote as $h \sim \exp (\mu)$.

\section{Success probability in the ideal channel}

The packet transmissions is successful if the receiving SINR at point $\mathrm{y}$ from a transmitter located at point $\mathrm{x}$ should be greater than or equal to the signal to interference plus noise SINR threshold $\gamma$. The success probability is denoted as

$$
\operatorname{Ps}(\gamma)=\mathrm{P}(\operatorname{SINR}>) \text {, }
$$

Where $\gamma$ is the SINR threshold.

$$
p_{s}=\exp \left(-\lambda_{P} C(\alpha)\left(\frac{\gamma}{u}\right)^{2 / \alpha} r^{2}\right) \text {. }
$$

\section{Throughput Analysis of Coexisting WBANs}

\section{Physical carrier sense of CSMA}

IEEE 802.15.4 MAC provides the capability to perform CCA in its CSMA-CA mechanism. Carrier sense is a fundamental mechanism in CSMA/CA protocols. Each user senses the channel before a transmission and defers the transmission if it senses a busy channel to reduce the collision. Use of the carrier sense option improves coexistence by allowing transmission back off if the channel is occupied by any other devices [14].

Physical carrier sense is a valid mechanism of CSMA-CA protocols to reduce collisions in WBANs coexistence, and the carrier sense range has a great influence on the network performance. Transmission range is around the core of sending node, but actually it is related to the receiver. The carrier sense range, however, is actually correlative with the transmission. The node need detect whether the channel is busy according to the carrier sense range threshold.

The unlicensed $2.4 \mathrm{GHz}$ the Institute for Supply Management (ISM) band is used by a variety of devices, standards and applications. A total of 16 channels are available in the $2.4 \mathrm{GHz}$ band of IEEE 802.15.4 standard, numbered 11 to 26 , each with a bandwidth of $2 \mathrm{MHz}$ and a channel separation of $5 \mathrm{MHz}$.

According to the carrier sense threshold $\beta$, the contention domain of a test CN located at a generic position $\mathrm{x} \in \mathbb{R}^{2}$ can be defined as:

$$
P_{t} h\|x-y\|^{-\alpha} \geq \beta .
$$

Without loss of generality, due to the static of the PPP model, we



Figure 1: WBANs coexistence. 
can take the node to be at the origin $\mathrm{o}$. Given that a receiver is located at origin $(0,0)$ and the distance between each transmitter and the origin is $x_{i}$, The contention domain radius:

$$
r_{0}=\left(\frac{P_{t} h}{\beta}\right)^{1 / \alpha},
$$

$r_{\mathrm{o}}$ is the carrier-sensing range.

\section{Carrier sense range optimization}

The carrier sense range $r_{\mathrm{o}}$ is a tunable design parameter that can seriously affect the performance of MAC layer. An optimal carrier sense range can balance the tradeoff between the throughput and the interference. The contention domain of each node will increase when the carrier sense threshold is diminished. Therefore, the number of WBANs, which transmit data on the same logical channel, will increase if the carrier sense threshold is decreased. However, it will increase interference and affect the outage probability. Clearly, the larger $r_{\mathrm{o}}$ is, the level of interference and the outage probability will increase. The actual channel throughput, however, does not necessary increase with $r_{\mathrm{o}}$, since a larger $r_{\mathrm{o}}$ also leads to a lower level of spectral efficiency. Considering all these factors, we will optimize the carrier sense threshold to increase the throughput as much as possible.

The interference will improve as the density of the network increases especially the effect of nearest node. For the reason that, we first consider the distance of the nearest point of the process of WBANs distribution. The distance denoted by $C$ from any node to its nearest node. We adjust the carrier sense range power according to the nearest distance when the $C$ is less than contain domain.

The nearest node distance distribution:

$$
f(C)=2 \pi \lambda_{p} C \exp \left(-\pi \lambda_{p} C^{2}\right),
$$

The average distance is

$$
\mathrm{E}[\mathrm{C}]=\int_{0}^{\infty} r \cdot 2 \lambda_{p} \pi r e^{\left(-\lambda_{p} \pi r^{2}\right)} d r=\frac{1}{2 \sqrt{\lambda_{p}}},
$$

According to $\left(\frac{P_{\mathrm{t}} \mathrm{h}}{\beta}\right)^{1 / \alpha}=\frac{1}{2 \sqrt{\lambda_{p}}}$,

The carrier sense threshold $\beta$ is expressed as $\beta=P_{t} h\left(2 \sqrt{\lambda_{p}}\right)^{\alpha}$.

We can express the throughput under a certain limit of success probability, which can be expressed as

maximize $S(\beta)$

subject to $p_{s}(\beta)>\sigma$

$$
\beta=P_{t} h\left(2 \sqrt{\lambda_{p}}\right)^{\alpha}
$$$$
\beta_{\min }<\beta<\beta_{\text {max }}
$$

where the $S(\beta)$ denotes the throughput of WBANs, $p_{s}(\beta)$ is success probability and $\sigma$ is minimum constraint value of success probability.

\section{The throughput of coexisting WBANs under HCPP model}

In CSMA, all the nodes in the network contend for the channel. The transmitter nodes can use the same channel to communicate at the same time in the coexisting WBANs, which resulted in the mutual interference of each other. It is a problem that how to control the interference in the networks. In order to reduce the intensity of interference signal effectively, the protection area in the model of WBAN is proposed. There will be a smaller exclusion zone around any receive node. In this area, no other transmitter node is allowed to transmit data, which can greatly reduce the interference. The guard zone size of influence of WBANs is optimized using stochastic geometry, which can decreases the interference and improve the throughput. This model is also known as the HCPP (Figure 2).

Each time a node wishes to transmit data frames or MAC commands, it first waits for a backoff period. If the channel is found to be idle, following the random backoff, the device transmits its data. If the channel is found to be busy following the random backoff, the node waits for another random period before trying to access the channel again [14]. Throughout this paper, we define the backoff time as a mark $m$. The node with the small marks will transmit its packet firstly. The model with guard zone retains only for the points having the lowest mark in their contention domains. Therefore the density of model with guard zone based on PPP model is given by ElSawy and Hossain [10]

$$
\lambda_{h}=\frac{\left(1-\mathrm{e}^{-\lambda_{p} \delta z^{2}}\right)}{\partial \mathrm{z}^{2}},
$$

where $\mathrm{z}$ is denoted as the size of guard zone (Figure 3). Around each transmit node, the left node has the minimum mark.

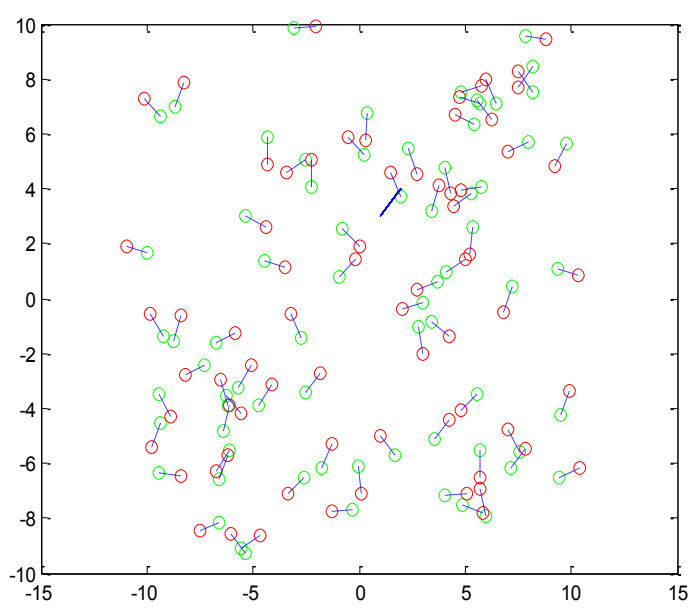

Figure 2: PPP model (Red dots represents the CNs while the green dots represents the TNs.).

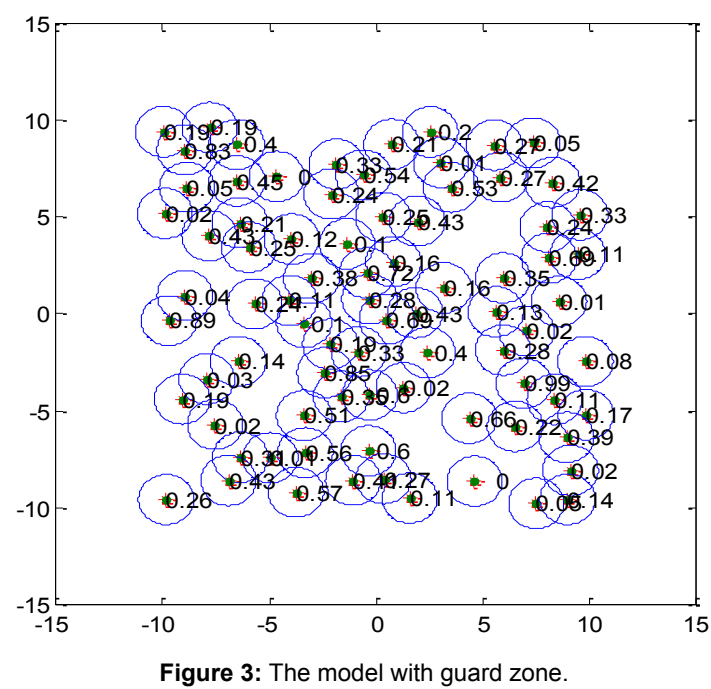

Figure 3: The model with guard zone. 
The throughput of a WBAN is defined as the maximum spatial intensity of successful transmissions in a certain domain under the condition that the outage probability does not exceed some specified threshold. Let $S$ is the normalized the coexist networks throughput, defined as the average time of successfully transmit payload bits

$$
S=\lambda_{h} p_{s} L
$$

where the $L$ is transmit rate. According to the success probability $P_{s}$, the throughput can be expressed as

$$
S=\lambda_{h} p_{s} L=\frac{\left(1-\mathrm{e}^{-\lambda_{p} \delta z^{2}}\right)}{\delta \mathrm{z}^{2}} \exp \left(-\lambda_{h} C(\alpha)\left(\frac{\gamma}{u}\right)^{2 / \alpha} r^{2}\right) L .
$$

We can get the following nonlinear optimization problem:

maximize $S(z)$

subject to $p s(\beta)>\sigma$,

Following the similar way of Lagrange duality approach, In particular, defining

$$
\mathrm{g}(\mathrm{z})=S(\mathrm{z})+\zeta\left(p_{s}(\beta, z)-\sigma\right.
$$

According to $d(g(z)) / d(z)$, the optimized value is obtained $z=0.36$.

\section{Performance Evaluation}

The aim of this section is to verify our throughput analysis through MATLAB simulations. PPP model have adopted for the operation of coexisting IEEE 802.15.4-based WBANs. We simulate the operation of WBANs in a static situation with changing density of coexisting WBANs and thinning the PPP model through adding a guard zone in each receiving node.

We consider that all nodes, including the $\mathrm{CN}$ and $\mathrm{TN}$, have the same transmit power equal to $-10 \mathrm{dBm}$. The effective transmission range will change with the changing of the carrier sense threshold. The contain domain is the carrier sense range of each transmitter. Many transmission nodes have distributed in the $20 \mathrm{~m}^{\star} 20 \mathrm{~m}$ area according to certain density. To simplify the analysis, we exploit the fact that, according to the CSMA standard, there is only one node sending data at the same time in each WBAN, such as Figure 2. The red dots represent the TNs while the green dots represent CNs in Figure 2. Figure 3 shows that the HCPP model with guard zone $0.5 \mathrm{~m}$. The HCPP model describes the patterns that only the minimal mark is retained in the threshold of the guard zone. The center of circle represents a TN while the decimal is the value mark. The radius of circle is the size of guard zone.

The success probability in PPP and HCPP is shown in Figures 4 and 5. Figure 4 illustrates the success probability with the changing carrier sense threshold $\gamma$ under the different coexistence density $\lambda_{p}$. It was concluded that the faster success probability decreases the higher the density. This is because, at higher sensing threshold, the transmission distance of TN is smaller. Figure 5 shows that the success probability of PPP model and HCPP model under the same density. The $p_{s}$ with guard zone is significantly higher than no guard zone. The success probability of HCPP is always higher than 0.9. However, the PPP model degrades quickly as the density increases. This suggests that the HCPP with guard zone model naturally decreases the interference. Therefore, the optimal guard zone can enhance the performance of coexisting networks.

Figure 6 shows that the success probability with the optimization according to the networks density, which is also in the light of the closest node's distance. The results indicate that the success probability is much better than the blue curve $\lambda_{p=1}$ in Figure 4. This is because, at lower density, we can enlarge the contention domain of each node. That is, the carrier sense threshold should be reduced. For this reason, conversely, the higher the density of WBANs coexistence, the carrier sense threshold should be increased. Therefore, we adjust the carrier sense range power according to the nearest distance.

The throughput simulation results are listed in Figures 7-9. The results of Figure 7 are consistent with the results of Figure 5. The throughput is higher because the networks success probability is large. The throughput in Figure 8 shows that the throughput will decrease when the network coexistence is greater than a certain density. This is because when the density is large, the conflict between each other has increased and the interference has become large. Figure 9 compares that the throughput under the different density $\lambda_{p}$. Especially when the network density is greater than a certain value, the throughput changed irregularly. The throughput is low when the size of guard zone is relatively small or very large. From this figure, we can achieve an optimal throughput at $0.3<$ zone $<0.4$.

\section{Conclusion}

WBANs coexistence may result in severe degradation in network performances. In this paper, we have analyzed the impact of channel sensing range. In addition, we model the system adopting the stochastic geometry Poisson point process and analyze the impact of guard zone

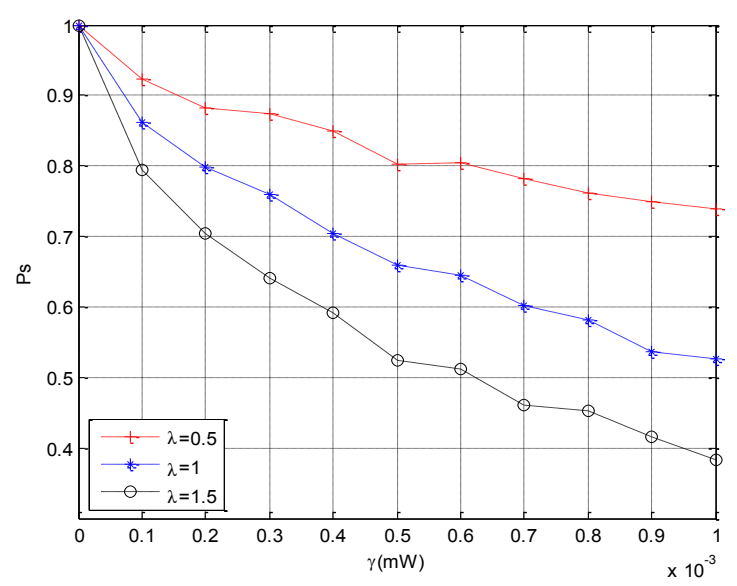

Figure 4: The success probability with the carrier sense threshold $y$.



Figure 5: The success probability with the guard zone. 
Citation: Wang Y, Liu R, Shu M, Chen C (2017) Stochastic Geometry Analysis of Throughput for Wireless Body Area Networks. Adv Robot Autom 6: 172. doi: 10.4172/2168-9695.1000172

Page 5 of 6

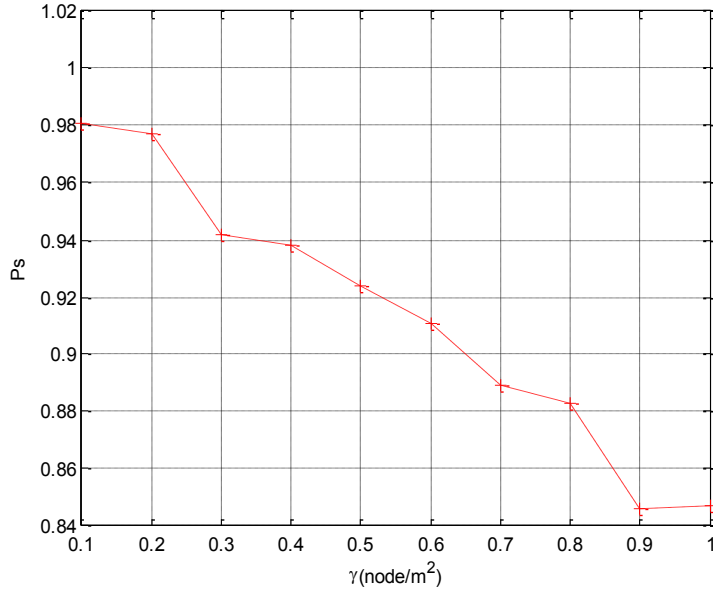

Figure 6: The success probability with the optimization according to the closest node's distance.



Figure 7: The success probability with the carrier sense threshold $\mathrm{y}$.

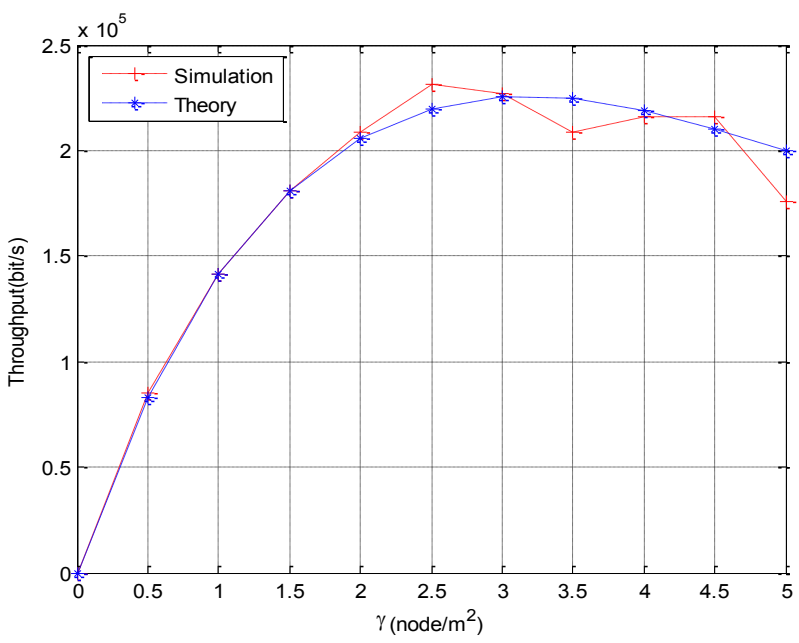

Figure 8: The throughput of PPP model with density $\gamma$.

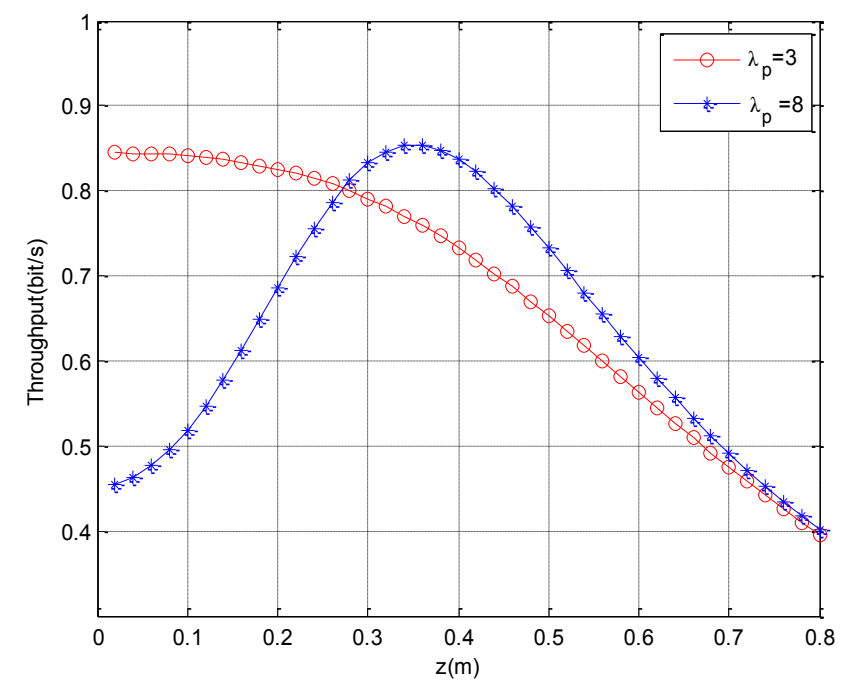

Figure 9: The throughput of HCPP model with guard zone.

size to networks performance. The results show that the protection of the area of the network can reduce the interference and reduce the available communication area. Too small to reduce the effect of the signal intensity of the interference signal is limited, and cannot effectively improve the network capacity of the network, the excessive protection of the regional radius will lead to a serious decrease in the available communication area in the network, but will eventually make the throughput decreased. Therefore, the size of guard zone is the key parameter in HCPP model. Finally, the optimal guard zone is obtained by the tradeoff between the guard zone and the throughput. Through theory analysis and simulations, we show that the guard zone can greatly reduce the interference in the network, increase the success probability, and reasonably set the size of the guard zone can reach the maximum the throughput. The energy and spectral efficiency, moreover, have been improved.

\section{Acknowledgments}

This work is supported by the National Natural Science Foundation of China under Grant No. 61401256, Natural Science Foundation of Shandong Province under Grant No. ZR2017MF029 and the Shandong Provincial Science and Technology Project under Grant No. 2014GSF118107.

\section{References}

1. Yang GZ (2006) Body Sensor Networks. London: Springer.

2. Cao H, Leung V, Chow C (2009) Enabling Technologies for Wireless Body Area Networks: A Survey and Outlook. IEEE Commun. Mag 47: 84-93.

3. Liu R, Wang Y, Wu S, Wang CX, Zhang W (2016) Energy efficiency and area spectral efficiency tradeoff for coexisting wireless body sensor networks. Science China Information Sciences 59: 1-15.

4. Baccelli F, Błaszczyszyn B (2010) Stochastic geometry and wireless networks Volume II Applications. Foundations and Trends in Networking 4: 1-312.

5. Zhao Z, Yang GH, Liu Q, Li VOK, Cui L (2011) Implementation and Application of a Multi-radio Wireless Sensor Networks Testbed. IET Wireless Sensor Systems 1: 191-199.

6. Won C, Youn JH, Ali HH, Sharif H, Deogun J (2005) Adaptive Radio Channel Allocation for Supporting Coexistence of 802.15.4 and 802.11b. IEEE Xplore 4: $2522-2526$

7. Yi P, Iwayemi A, Zhou C (2011) Developing ZigBee Deployment Guideline under Wi-Fi Interference for Smart Grid Applications. IEEE Transactions on Smart Grid 2: 110-120.

8. EISawy H, Hossain E, Camorlingga S (2014) Spectrum-Efficient Multi-Channe 
Citation: Wang Y, Liu R, Shu M, Chen C (2017) Stochastic Geometry Analysis of Throughput for Wireless Body Area Networks. Adv Robot Autom 6: 172. doi: 10.4172/2168-9695.1000172

Page 6 of 6

Design for Coexisting IEEE 802.15.4 Networks: A Stochastic Geometry Approach. IEEE Transactions on Mobile Computing 13: 1611-1624.

9. Xu K, Gerla M, Bae S (2002) How effective is the IEEE 802.11 RTS/CTS handshake in ad hoc networks, in Proc. IEEE GlobeCom.

10. EISawy H, Hossain E (2013) A modified hard core point process for analysis of random CSMA wireless networks in general fading environments. IEEE Trans. Commun. 61: 1520-1534.
11. Muhlethaler $P$, Najid A (2003) Throughput optimization of a multihop CSMA mobile ad hoc network, INRIA.

12. Liu R, Wang Y, Shu M, Wu S (2017) Throughput assurance of wireless body area networks coexistence based on stochastic geometry. PloS One 12: 1-22.

13. Deylami M, Jovanov E (2012) Performance Analysis of Coexisting IEEE 802.15.4-Based Health Monitoring WBANs. IEEE EMBS.

14. (2006) Wireless Medium Access Control (MAC) and Physical Layer (PHY) Specifications for Low-Rate Wireless Personal Area Networks (WPANs). 